\title{
Method for the reduction of leakage in labyrinth seals by adapting the seal geometry to match the flow conditions
}

\author{
Damian Joachimiak ${ }^{1^{*}}$ \\ ${ }^{1}$ Institute of Thermal Engineering, Poznan University of Technology, 60-965 Poznan, Poland
}

\begin{abstract}
In this paper a method for reducing leakage in labyrinth seals is presented. This method is based on CFD calculations and consists in the analysis of the phenomenon of gas kinetic energy carry-over in chambers of the seal between gaps. It belongs to the group of geometrical inverse problems and is designed for seals of given outside dimensions. For straight through labyrinth seals it enables determining the number of teeth and their optimal arrangement. This method was developed based on numerical and experimental tests. Examples of numerical calculations presented in this paper prove that this method is effective for straight through seals. We obtained the reduction of leakage ranging from 8.7 to $9.4 \%$ relative to the initial geometry with no change in the outside dimensions of the seal.
\end{abstract}

\section{Introduction}

Labyrinth seals are widely applied in a variety of fluidflow machines such as steam turbines, gas turbines, compressors and blowing machines. Such seals reduce leakage in the space between, for example, stationary body and the rotating shaft or its elements such as the shroud of the blade ring. Labyrinth seals are highly impactful on the efficiency of high power machines. Straight through labyrinth seals are applied in steam turbines in spots far from the thrust bearings [1-3]. Inverse problems are widely applied to solve engineering problems [4-12]. In this paper the inverse problem related to the choice of the seal geometry to obtain minimal leakage is presented.

Labyrinth seal prevent the leakage between two spaces of different gas pressure $p_{\text {in }}>p_{\text {out }}$ (fig. 1). Due to the differential pressure gas flows in the labyrinth seal. Labyrinth seal operates in such a way that the flowing gas expands sequentially in gaps (fig. 1). In these places the gas pressure energy changes into the kinetic energy which results in a significant drop of the gas pressure in the region of the clearance (fig. 2a). In chambers downstream clearances occurs the dissipation of the gas kinetic energy to the heat as the result of the flow turbulence caused by static stresses between the flowing gas and the walls as well as by gas rotational movement.

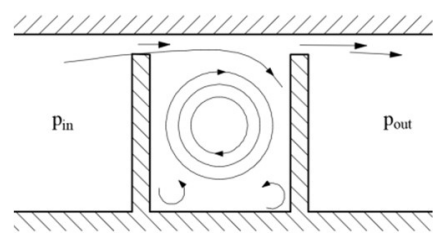

Fig. 1. Illustrative geometry of the straight through seal with marked gas flow

\footnotetext{
* Corresponding author: damian.joachimiak@put.poznan.pl
}

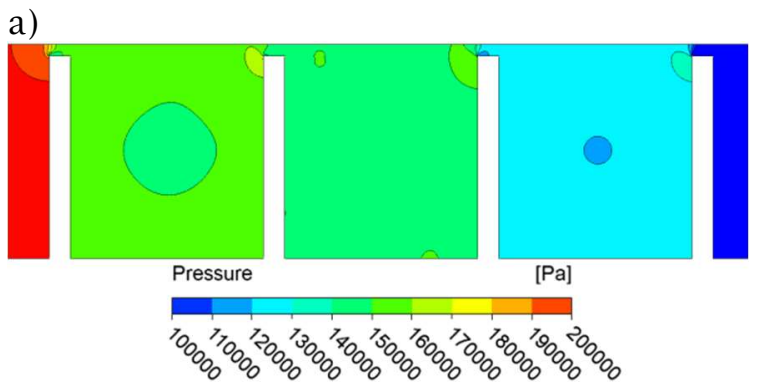

b)

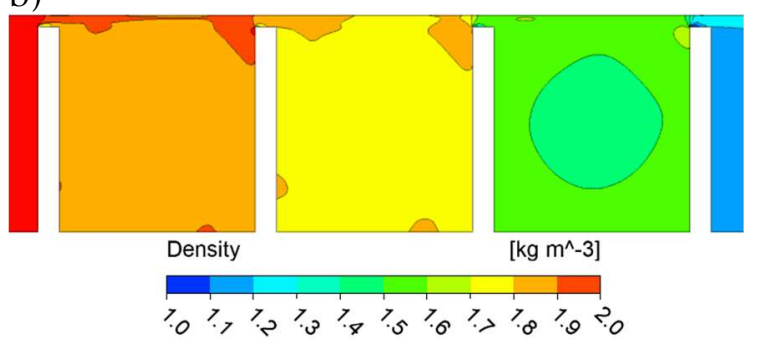

Fig. 2. Selected distribution of: a) the pressure, b) the gas density in the straight through seal for the pressure ratio $p_{\text {in }} / p_{\text {out }}=2$

Generally, working medium flowing through the labyrinth seal is gas. In segments of the seal characterized by significant drop in pressure $\left(p_{\text {in }} / p_{\text {out }} \geq 2\right)$ (fig. 2a) there occurs a noticeable change in gas density (fig. 2.b). From the gas flow continuity equation described by the dependence

$$
\dot{m}=A \rho c
$$

where: $A$ - denotes the flow field in the clearance, $\rho-$ density, $c$ - gas velocity, it arises that the velocity of gas expanding while flowing through the gap increases (fig. 3 ). Labyrinth seals applied today usually have chambers 
of fixed dimensions. As a result of expanding gas flow in the seal between gaps, changing flow conditions occur there. In chambers occurs a non-uniform reduction of the gas velocity (fig. 5). This phenomenon was described in reference paper [13].

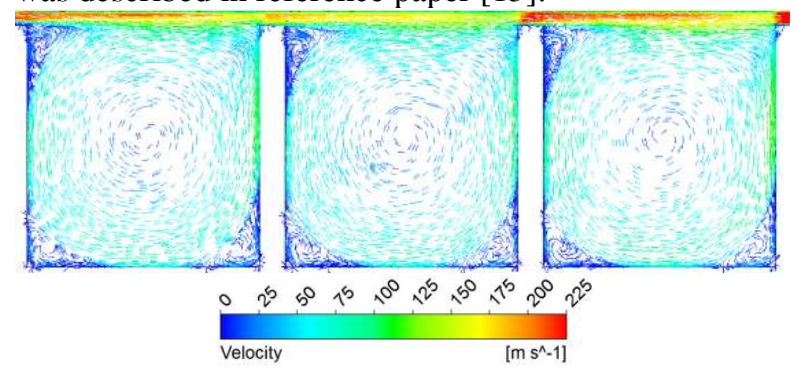

Fig. 3. Vectors of velocity fields in a chosen segment

The leakage in seals is liable to the phenomenon of gas kinetic energy carry-over occurring between clearances (fig 3). This phenomenon is particularly intensive in straight through seals where the expanding gas stream flows through the whole chamber length with a high velocity. The degree of seal wear has considerable impact on the leak-tightness of the seal [14-16]. Leakage of the seal depends on such features of the seal geometry as pitch, length, and height of the chamber [17-20].

In paper [13] the relation between the increased number of teeth along the uniform length of the straight through seal and the leakage was analysed. It arises from data included in that paper that for the straight through seal there exists a range of seal pitch for which the smallest leakage was achieved. Moreover, it was proved that if too many teeth were applied the leakage in the seal increased. For a small tooth pitch, the nature of the gas flow in a straight-through seal is similar to that in a slotted seal [21]. Paper [22] presents the method for optimization of the stepped labyrinth seal with two teeth. Leakage reduction was obtained using CFD solver implemented to the TASCflow3 environment and Annealing optimization method. The place where the seal should be applied is usually determined at the stage of the flow machine design. For the given length and nominal size of the seal radial clearance the geometry of clearance characterized by minimal leakage is crucial.

\section{Optimization method}

Labyrinth seals designed nowadays have unchangeable geometry (lengths of chambers are the same) or sequentially repeated geometries of two or three chambers along the whole length of the seal segment. Sequentially repeated geometry results from technological conditions of producing the seal and the seal operation reliability. The method described below is dedicated for straight through labyrinth seals of the given length and inside and outside diameters. Leakage minimization is done by proper arrangement of seal teeth what means obtaining chambers of variable length.

\subsection{Choice of the initial geometry of the straight through seal for the method of optimization}

The initial geometry of the straight through seal (fig. 4) is characterized by the diameter $D$, height $H$, segment length $L S$ and pitch length $L P$, tooth thickness $B$ and the radial clearance $R C$. Geometries of straight through labyrinth seals used so far usually have the pitchchamber height ratio approximately equal to unity $(L P / H \approx 1)$. From the paper [15] it arises that leakage in straight through seals is significantly lower when the geometry of the seal is characterized by the parameter $(L P-B) / H$ approximately equal to 0.5 . The value of the parameter $(L P-B) / H$ is verified for the initial geometry. If its value differ significantly from the value of 0.5 then the number of seal teeth distributed uniformly along the given length LS is chosen in such a way to achieve ( $L P$ $B) / H \approx 0.5$. And this geometry is used as the initial geometry for the optimization method.

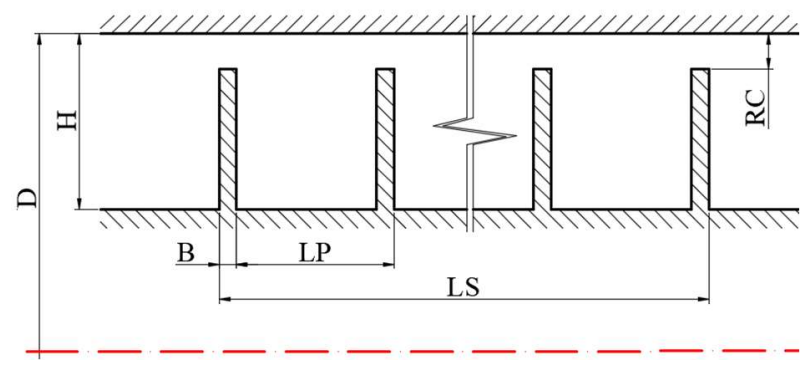

Fig. 4. Illustrative geometry of the straight through seal

\subsection{Assumptions for CFD calculations}

The geometry which is analysed is an axially symmetric geometry of 2D type. In the boundary layer minimum 20 grid cells were assumed. Calculations included $k-\omega$ SST turbulence model. In the boundary layer, the condition that $\mathrm{y}^{+}<2$ was kept. This paper presents results of stationary calculations of RANS type, including mass, momentum and energy conservation equations.

\subsection{Method for choosing the optimal pitch}

At the inlet to the seal the total pressure $p_{\text {in }}$ is known, and downstream the segment known is the static pressure $p_{\text {out. }}$ Based on these boundary conditions and the known initial geometry CFD calculations are performed. The method is based on the analysis of the distribution of the gas kinetic energy $E(r, x)$ in the axial direction (direction of the main flow) in the particular region of the seal. Gas kinetic energy in the axial direction is defined as

$$
E(r, x)=\frac{u^{2}}{2}
$$

where $u$ - the gas velocity into the axial direction. Method of adapting the geometry depending on flow conditions comprises in searching for local maximums of the non-dimensional gas kinetic energy into the axial direction $e_{\max }(\mathrm{i})$ described by the following formula 


$$
e_{\text {max }}(i)=\frac{E_{\text {max }}(i)-E_{\min }}{E_{\text {max }}-E_{\text {min }}}
$$

Lengths of the seal chambers depend on the distribution of local maximums of the kinetic energy along the whole length of the seal segment.

\section{Results of research}

The initial geometry is the segment of the straight through seal of the diameter $D=150 \mathrm{~mm}$, segment length $L S=61 \mathrm{~mm}$, segment height $H=10 \mathrm{~mm}$, pitch length $L P=10 \mathrm{~mm}$, tooth thickness $B=1 \mathrm{~mm}$ and the radial clearance $R C=0.4555 \mathrm{~mm}$ (fig. 5). This geometry has seven teeth and is characterized by the parameter $(L P-B) / H$ equal to 0.9 .

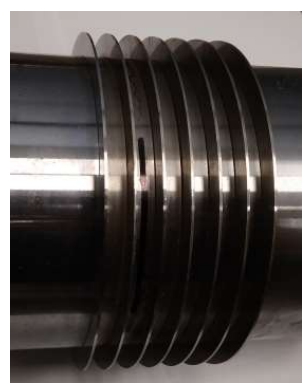

Fig. 5. Initial geometry of the straight through seal used for experimental tests

As a result of applying the optimization method, for the seal initial geometry a new geometry of 11 teeth and a variable pitch length $L P(i)$ was obtained (fig. 6).

As a result of applying the method for the leakage reduction, a high variability of pitch length $L P(i)$ (fig. 7) was obtained. The shortest, third, pitch in the segment is of $4.39 \mathrm{~mm}$, while the last pitch is the longest one with length of $9.96 \mathrm{~mm}$.

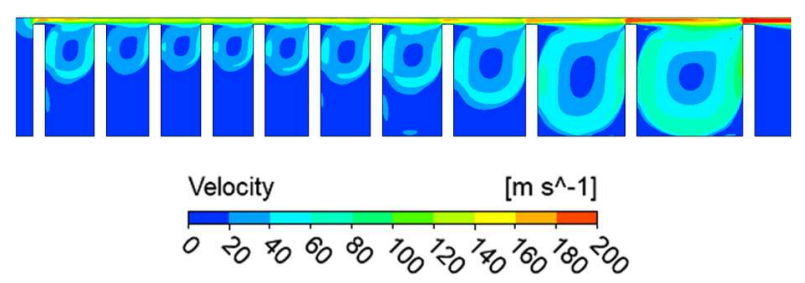

Fig. 6. Distribution of the gas velocity in the resultant geometry

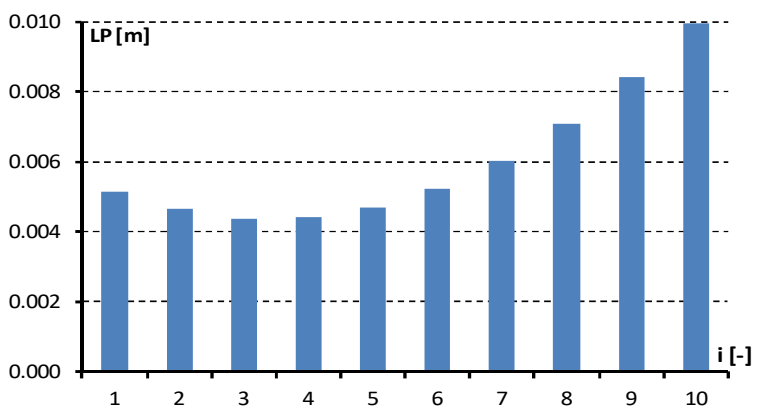

Fig. 7. Change of the pitch length $L P(i)$ of the resultant geometry of the straight through seal
Figure 7 indicates that the change of geometry done based on the method for the leakage reduction causes a change of the obtained gas velocity field in both, gaps and chambers of the seal. From the initial geometry a changed number of teeth - places of expansion with matching variable pitch for distributing the gas kinetic energy along the whole segment length is obtained. It means that in regions where relatively small gas kinetic energy is observed pitches of the seal are the shortest ones which in turn results in concentration of places of expansion. Where the kinetic energy increases, the seal pitches $L P(i)$ are proportionally greater. It arises from figure 6 that proper choice of pitch allows obtaining proper lengths of the seal chambers to achieve the maximum gas swirl.

For the initial geometry (fig. 5) and for the optimized geometry of the straight through seal (fig. 6) CFD calculations were performed for various pressure pin/pout ratios within the range from 2 to 2.8 . Results are summarized in table 1 including the leakage, the absolute difference and the relative difference of leakage for $p_{\text {out }} \approx 10^{5} \mathrm{~Pa}$.

Table 1. Change of the leakage depending on the pressure $\mathrm{p}_{\text {in }} / \mathrm{p}_{\text {out }}$ ratio for $p_{\text {out }} \approx 10^{5} \mathrm{~Pa}$ for the initial geometry and for the optimized one of the straight through seal

\begin{tabular}{|c|c|c|c|c|}
\hline $\begin{array}{c}p_{\text {in }} / p_{\text {ou }} \\
\mathrm{t} \\
{[-]} \\
\end{array}$ & $\begin{array}{c}\dot{m}[\mathrm{~kg} / \mathrm{s}] \\
\text { 7t_LS61_LP1 } \\
0\end{array}$ & $\begin{array}{c}\dot{m}[\mathrm{~kg} / \mathrm{s}] \\
\text { 11t_LS61_LPo } \\
\text { pt }\end{array}$ & $\begin{array}{c}\Delta \dot{m} \\
{[\mathrm{~kg} / \mathrm{s}]}\end{array}$ & $\begin{array}{c}\delta \dot{\boldsymbol{m}} \\
{[\%]}\end{array}$ \\
\hline 2 & 0.0440 & 0.0401 & $\begin{array}{c}0.003 \\
8\end{array}$ & 8.7 \\
\hline 2.4 & 0.0556 & 0.0504 & $\begin{array}{c}0.005 \\
2\end{array}$ & 9.4 \\
\hline 2.8 & 0.0663 & 0.0604 & $\begin{array}{c}0.005 \\
9\end{array}$ & 8.9 \\
\hline
\end{tabular}

a)

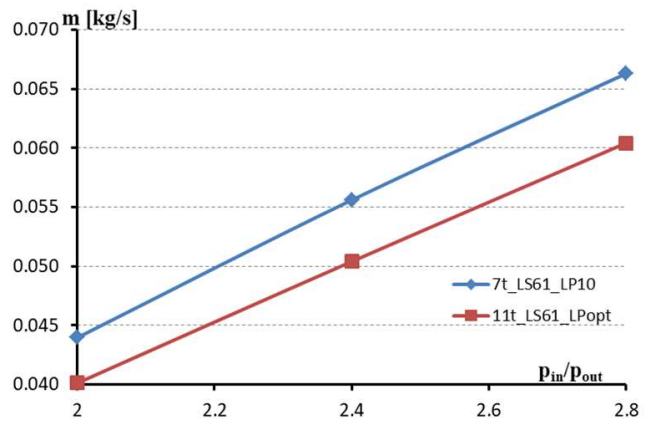

b)

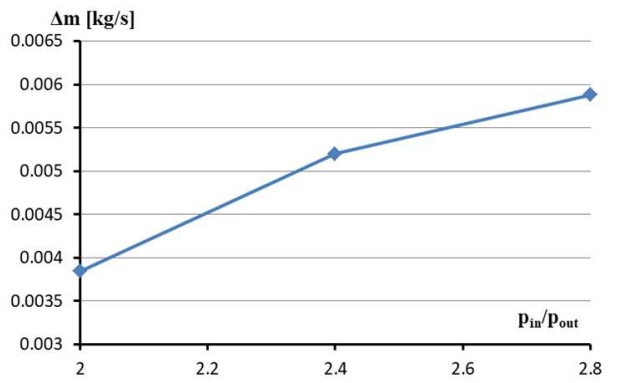

Fig. 8. Leakage as the function of pressure $p_{\text {in }} / p_{\text {out }}$ ratio: a) in the seal of the initial geometry 7t_LS61_LP10 and of the 
resultant one 10t_LS61_LPopt, b) the obtained value of the reduced leakage $\Delta \dot{m}$

For the increasing pressure $p_{\text {in }} / p_{\text {out }}$ ratio within the range from 2 to 2.8 , this method allows for obtaining increasing leak-tightness $\Delta \dot{m}$ within the range from 0.0038 to $0.0059 \mathrm{~kg} / \mathrm{s}$, which gives the relative leakage reduction $\delta \dot{m}$ in the range from $8.7 \%$ to $8.9 \%$ (fig. 8 , table 1).

\section{Conclusion}

Minimization of leakage in labyrinth seals is an important issue since it allows the efficiency of highpower flow machines to be improved. In this paper we presented some research on the reduction of leakage in the seal without changing its outside dimensions. Described method anticipates only changes in the geometry of inner elements of the seal. It is dedicated to geometry of axisymmetric seals. This method is based on a direct observation of real thermodynamic and flow conditions occurring in labyrinth seals. Adapting of the seal geometry to flow conditions allows for optimizing seals in fluid-flow machines such as steam turbines or compressors either being newly designed or being already installed but modernized or repaired now.

\section{Nomenclature}

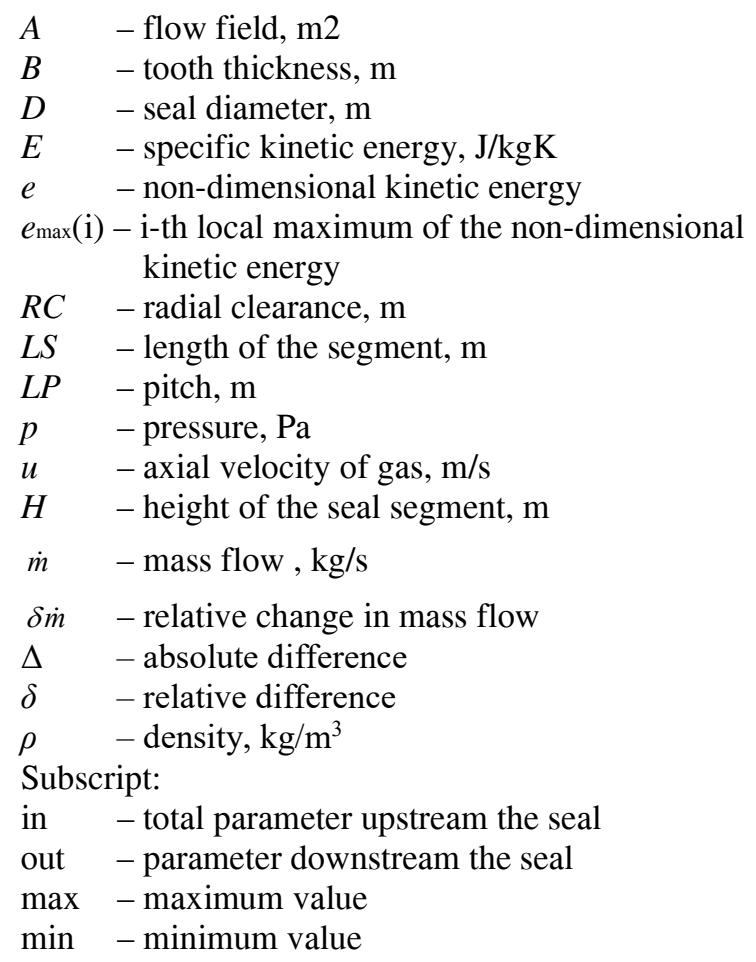

\section{References}

1. K. Trütnovsky, Berührungsfreie Dichtungen, Grundlagen und Anwendungen der Strömung durch
Spalte und Labyrinthe (Verlag des Vereins Deutscher Ingenieure, Düsseldorf, 1964)

2. P. R. N. Childs, Mechanical Design Engineering Handbook (Elsevier, 2019)

3. D. Joachimiak, and P. Krzyślak, Arch. Thermodyn. 36, 61 (2015)

Comparison of results of experimental research with numerical calculations of a model one-sided seal, Vol. 36, No. 2, 2015, 61-74.

4. K. Grysa, A. Maciąg, and A. Pawińska, Int. J. Heat Mass Transf. 55, 7336 (2012)

5. M. Joachimiak, Energies 14, 2903 (2021)

6. M. Joachimiak, Int. J. Numer. Methods Heat Fluid Flow 30, 4475 (2020)

7. M. Joachimiak, and M. Ciałkowski, Arch. Thermodyn. 39, 25 (2018)

8. M. Joachimiak, M. Ciałkowski, and Bartoszewicz J., Arch. Thermodyn. 35, 37 (2014)

9. M. Joachimiak, M. Ciałkowski, and A. Frąckowiak, Int. J. Numer. Methods Heat Fluid Flow 30, 1441 (2020)

10. M. Joachimiak, D. Joachimiak, M. Ciałkowski, L. Małdziński, P. Okoniewicz, and K. Ostrowska, Int. J. Therm. Sci. 145, 105985 (2019)

11. B. Maciejewska and M. Piasecka, Int. J. Heat Mass Transf. 107, (2017)

12. J. Taler, B. Weglowski, and M. Pilarczyk, Int. J. Numer. Methods Heat Fluid Flow 27, (2017)

13. D. Joachimmiak and P. Krzyslak, J. Appl. Fluid Mech. 12, 921 (2019)

14. D. Joachimiak and P. Krzyślak, Polish Marit. Res. 24, 83 (2017)

15. D. Joachimmiak, J. Appl. Fluid Mech. 13, 935 (2020)

16. D. Joachimiak and A. Frąckowiak, Energies 13, 5794 (2020)

17. J. Yang, F. Zhao, M. Zhang, Y. Liu, and X. Wang, Comput. Model. Eng. Sci. 126, 935 (2021)

18. W. Zhou, Z. Zhao, Y. Wang, J. Shi, B. Gan, B. Li, and N. Qiu, Alexandria Eng. J. 60, 3177 (2021)

19. X. J. Sun, P. Y. Song, X. P. Hu, W. Y. Mao, Q. G. Deng, and H. J. Xu, J. Appl. Fluid Mech. 14, (2021)

20. H. Qin, D. Lu, D. Zhong, Y. Wang, and Y. Song, Ann. Nucl. Energy 135, 106964 (2020)

21. D. Joachimiak, and P. Krzyślak, Arch. Thermodyn. 37, 95, (2016)

22. V. Schramm, J. Denecke, S. Kim, and S. Wittig, Int. J. Rotating Mach. 10, 365 (2004)

23. F. De Lillo, F. Cecconi, G. Lacorata, A. Vulpiani, EPL, 84 (2008)

24. L. T. De Luca, Propulsion physics (EDP Sciences, Les Ulis, 2009)

25. G. Plancque, D. You, E. Blanchard, V. Mertens, C. Lamouroux, Role of chemistry in the phenomena occurring in nuclear power plants 
circuits, in Proceedings of the International

Congress on Advances in Nuclear Power Plants,

ICAPP, 2-5 May 2011, Nice, France (2011) 